\title{
Solution-Processed Halide Perovskite Single Crystals with Intrinsic Compositional Gradients for X-Ray Detection
}

Xin Wang*, ${ }^{\dagger}$, Yuwei Li ${ }^{\dagger}$, Yubing Xu+, Yuzhu Pan ${ }^{\dagger}$, Chongyang Zhu, Dianjia Zhu ${ }^{\dagger}, Y^{\dagger}$ a Wu ${ }^{\dagger}$, Guanwen $\mathrm{Li}^{\dagger}$, Qi Zhang ${ }^{\dagger}$, Qing $\mathrm{Li}^{\dagger}$, Xiaobing Zhang ${ }^{\dagger}$, Jun $\mathrm{Wu}^{\dagger}$, Jing Chen ${ }^{\dagger}$ and Wei Lei*, ${ }^{\dagger}$

†oint International Research Laboratory of Information Display and Visualization, School of Electronic Science and Engineering, Southeast University, Nanjing 210096, China

$\$$ SEU-FEI Nano-Pico Center, Key Laboratory of MEMS of the Ministry of Education, Southeast University,

Nanjing 210096, China 


\section{Supplementary Materials.}

\section{Supporting Figures:}
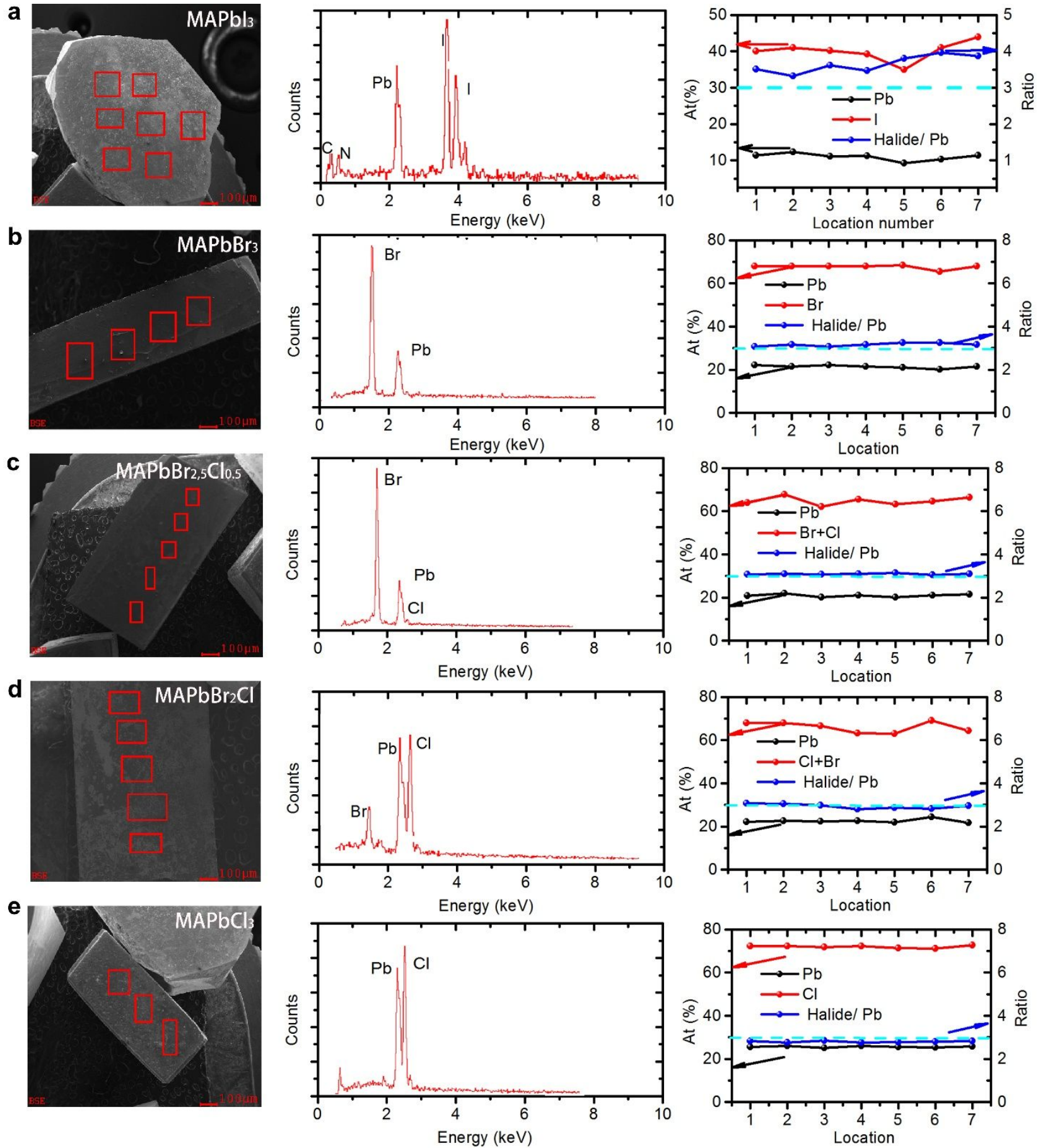

Figure S1. EDX results for OPSCs with different halide components. (a) SEM photo, EDX spectrum and elemental atomic ratios of the $\mathrm{MAPbI}_{3}$ OPSC. (b) SEM photo, EDX spectrum and elemental atomic ratios of the MAPbBr ${ }_{3}$ OPSC. (c) SEM photo, EDX spectrum and elemental atomic ratios of the $\mathrm{MAPbBr}_{2.5} \mathrm{Cl}_{0.5}$ OPSC. (d) $\mathrm{SEM}$ photo, EDX spectrum and 
elemental atomic ratios of the $\mathrm{MAPbBrCl}_{2}$ OPSC. (e) SEM photo, EDX spectrum and elemental atomic ratios of the $\mathrm{MAPbCl}_{3}$ OPSC.

The atom percentages of $\mathrm{C}, \mathrm{Cl}, \mathrm{Br}$ and $\mathrm{Pb}$ in $\mathrm{MAPbBr}_{2.5} \mathrm{Cl}_{0.5}$ OPSC are 15.09, 11.34, 52.15 and 21.42 respectively. 

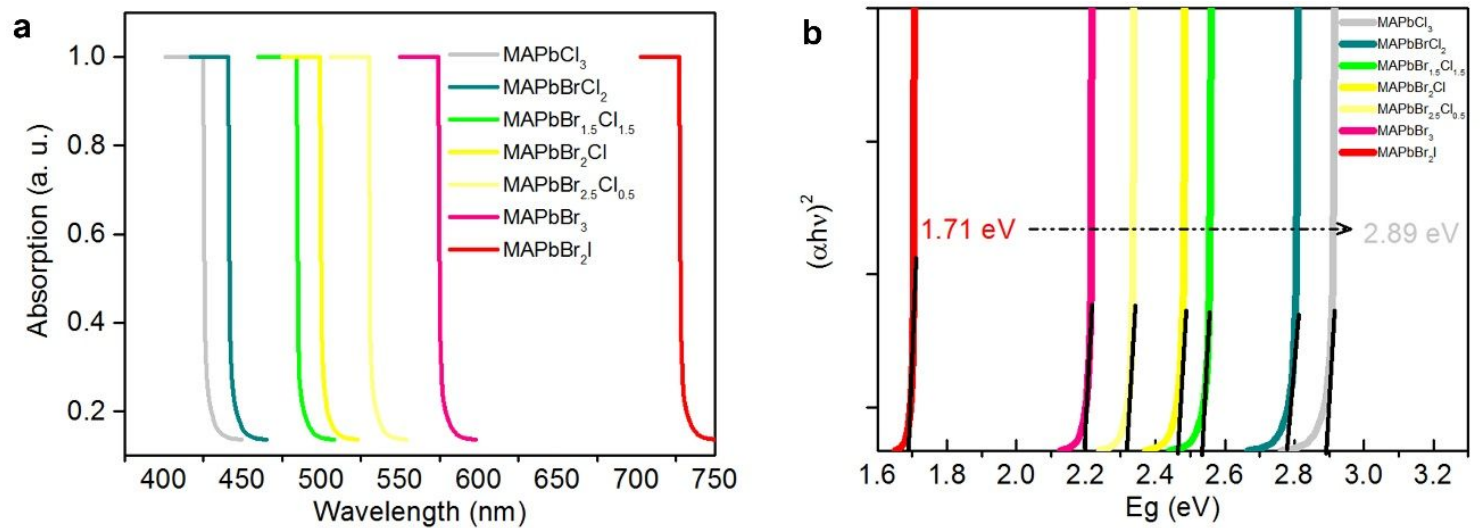

C
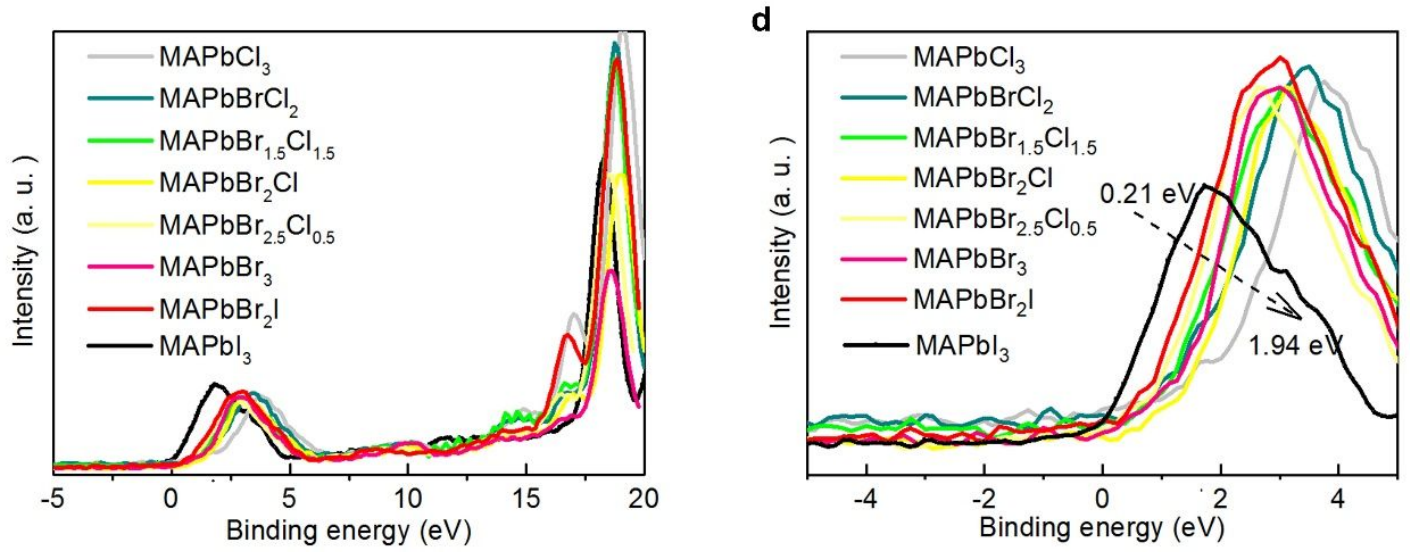

e
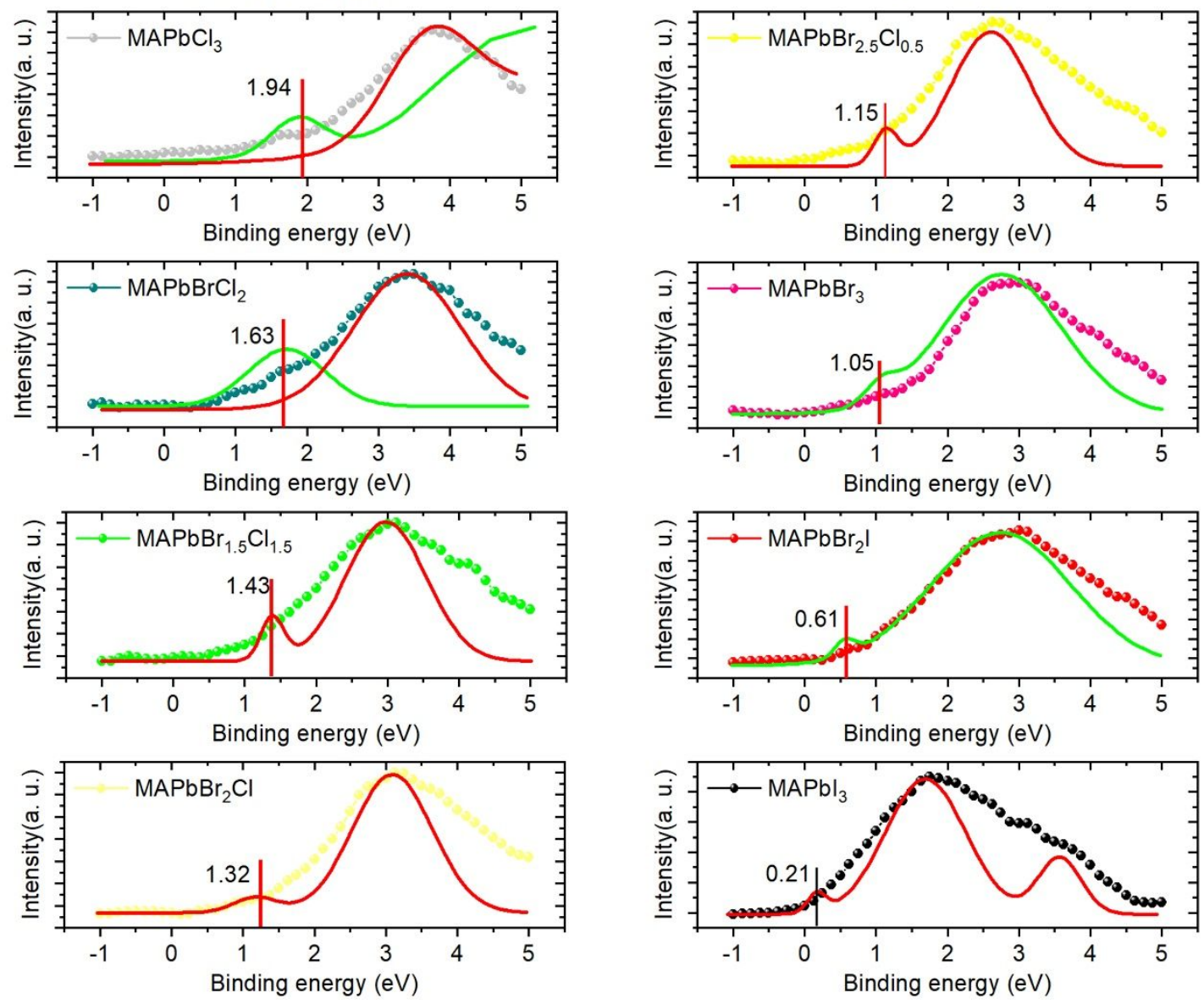
Figure S2. Absorption spectra and X-ray photoelectron spectroscopy (XPS) results for OPSCs with different halide components. (a) Absorption spectra. (b) Tauc plots. (c) Longrange XPS results from $-5 \mathrm{eV}$ to $20 \mathrm{eV}$. (d) Detailed XPS results for the valence band maxima. (e) Fitting plots of XPS results for valence band maxima. 


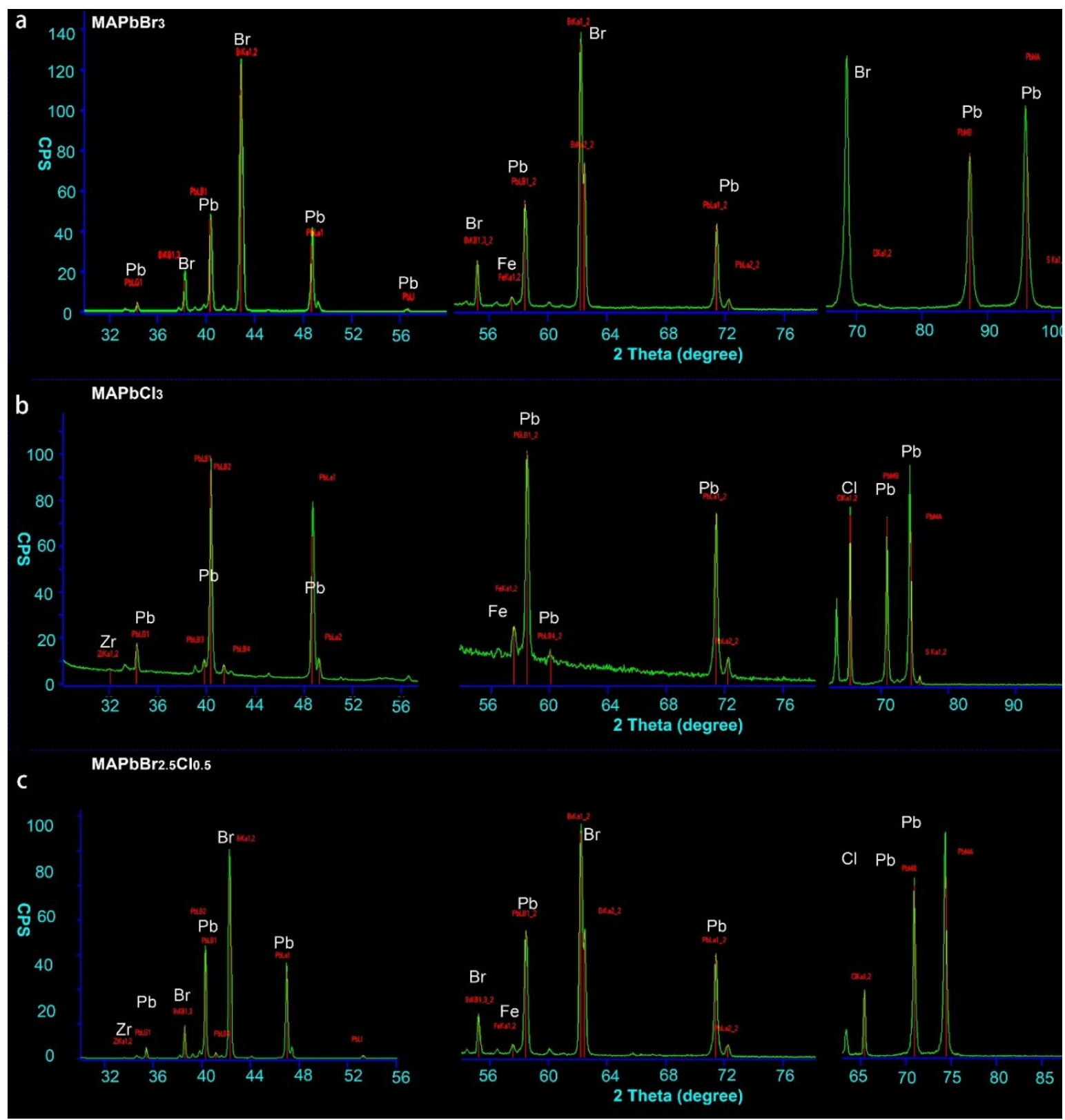

Figure S3. X-ray fluorescence spectrometer. (a) $\mathrm{MAPbBr}_{3} \mathrm{OPSC}$. (b) $\mathrm{MAPbCl}_{3} \mathrm{OPSC}_{\text {. }}$ (c) $\mathrm{MAPbBr}_{2.5} \mathrm{Cl}_{0.5} \mathrm{OPSC}$

\begin{tabular}{|l|c|c|c|c|c|}
\hline & $\mathrm{Br}(\mathrm{AT} \%)$ & $\mathrm{Cl}(\mathrm{AT} \%)$ & $\mathrm{Pb}(\mathrm{AT} \%)$ & Impurity(ppm) & Method \\
\hline $\mathrm{MAPbCl}_{3}$ & 0 & 72.49 & 27.5 & $18(\mathrm{Fe})$ & XFS \\
& 0 & 71.37 & 28.63 & --- & EDX \\
\hline $\mathrm{MAPbBr}_{2.5} \mathrm{Cl}_{0.5}$ & 60.1 & 14.8 & 25.1 & $15(\mathrm{Fe}, \mathrm{Zr})$ & XFS \\
& 61.4 & 13.4 & 25.2 & --- & EDX \\
\hline $\mathrm{MAPbBr}_{3}$ & 77.9 & 0 & 22.1 & $22(\mathrm{Fe}, \mathrm{Si})$ & XFS \\
& 75.56 & 0 & 24.44 & --- & EDX \\
\hline
\end{tabular}

Table S1. Element results of X-ray fluorescence. 


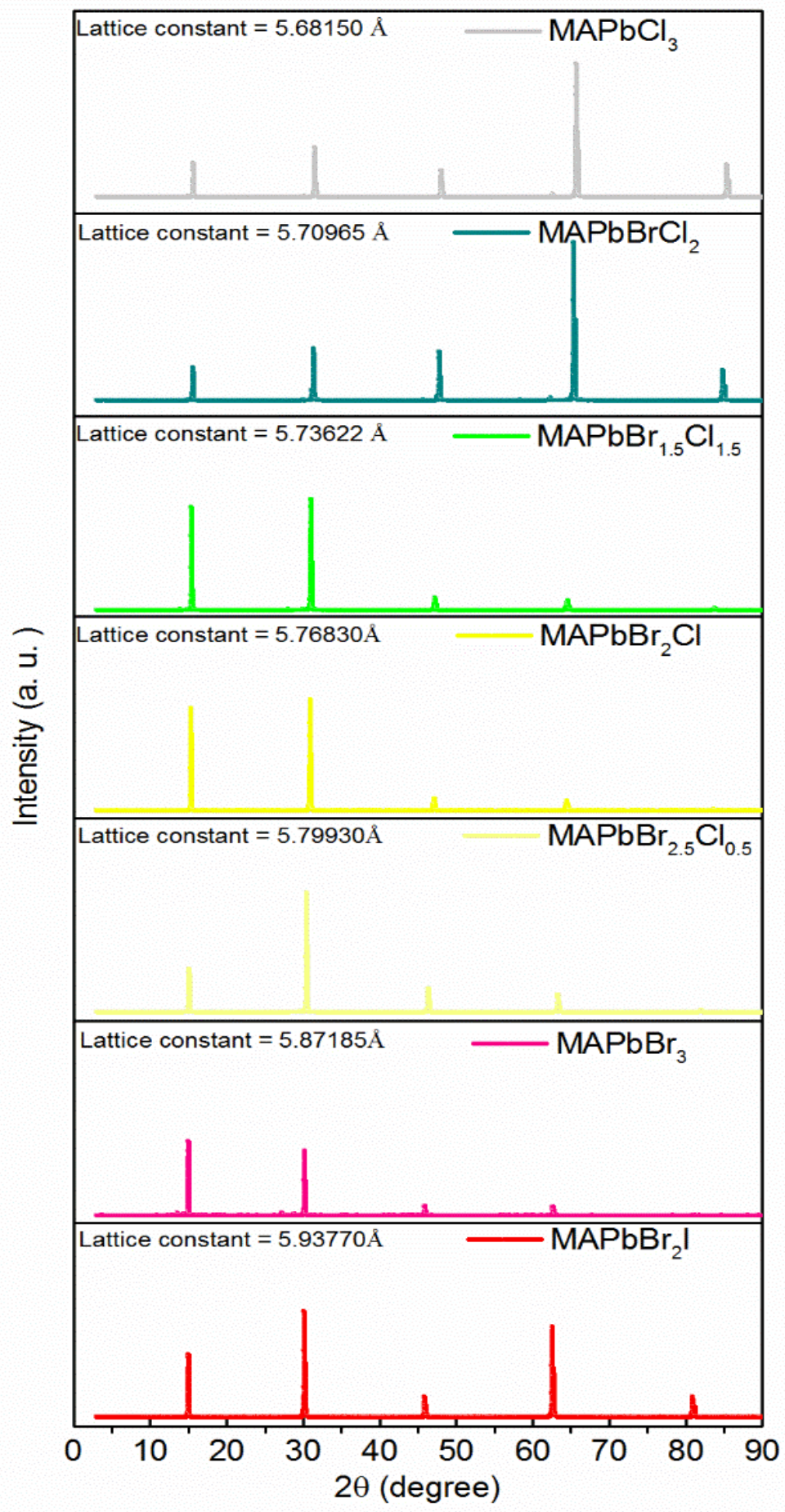

Figure S4. X-ray diffraction results for OPSCs with different halide components. 


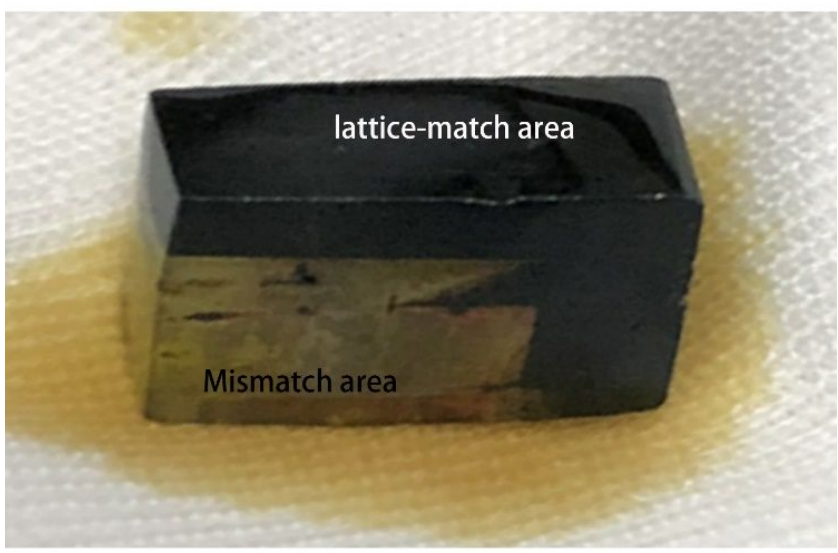

Figure S5. Lattice constant selected $\mathrm{SPE}$. The $\mathrm{MAPbI}_{3} \mathrm{OPSC}$ can only grow on $\mathrm{MAPbBr}_{2} \mathrm{I}$ and is unable to grow on the $\mathrm{MAPbBr}_{\mathrm{x}} \mathrm{Cl}_{(3-\mathrm{x})}$ OPSC 

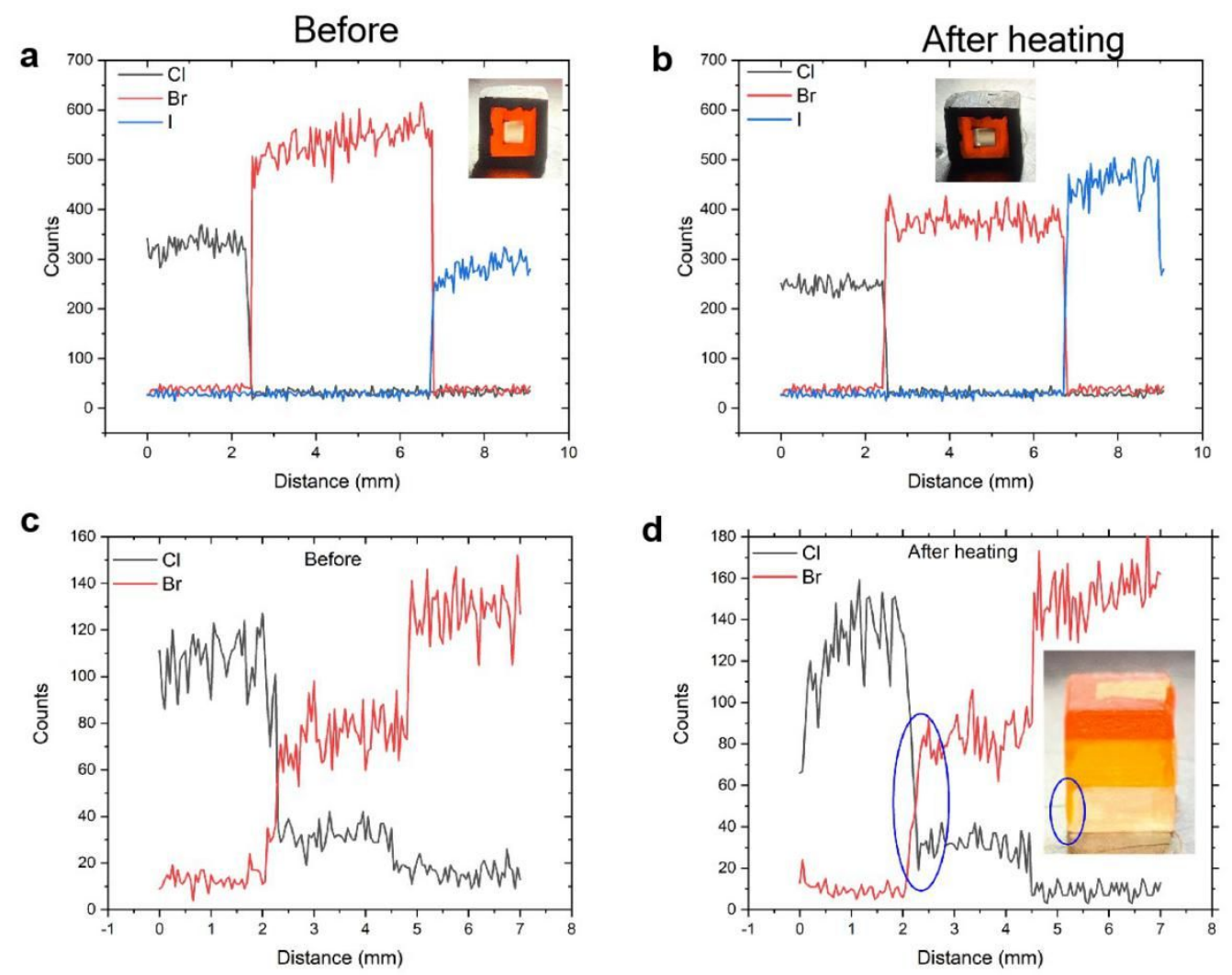

Figure S6. Line scan of cascade OPSCs. (a) $\mathrm{MAPbCl}_{3} / \mathrm{MAPbBr}_{3} / \mathrm{MAPbI}_{3}$ before heating. (b) $\mathrm{MAPbCl}_{3} / \mathrm{MAPbBr}_{3} / \mathrm{MAPbI}_{3}$ after heating. (c) $\mathrm{MAPbCl}_{3} / \mathrm{MAPbBr}_{2.5} \mathrm{Cl}_{0.5} / \mathrm{MAPBr}_{3}$ before heating. (d) $\mathrm{MAPbCl}_{3} / \mathrm{MAPbBr}_{2.5} \mathrm{Cl}_{0.5} / \mathrm{MAPBr}_{3}$ after heating.

To figure out the halide ions interdiffusion in cascade OPSCs, we use two different cascade OPSCs for investigation. Before the discussion, we think that there is no doubt that the existence of halide ions interdiffusion inside cascade OPSCs. And the halide ions would be the same as a halide composition gradient after a long time. Here, we focus on how to slow down the speed of halide ions interdiffusion and maintain the junctions.

In first experiment, we use a $\mathrm{MAPbCl}_{3} / \mathrm{MAPbBr}_{3} / \mathrm{MAPbI}_{3}$ structure of cascade OPSC and heat it at $100^{\circ} \mathrm{C}$ for 6 hours. And the line scan mode in EDX is used to measure the halides distribution before/after heating. As shown in Figure S7a and Figure S7b, the chlorine-bromide ions diffusion 
could be found near the interface of $\mathrm{MAPbCl}_{3} / \mathrm{MAPbBr}_{3}$ and the iodide-bromide ions diffusion is negligible between $\mathrm{MAPbBr}_{3} / \mathrm{MAPbI}_{3}$ 。

In second experiment, we use a $\mathrm{MAPbCl}_{3} / \mathrm{MAPbBr}_{2.5} \mathrm{Cl}_{0.5} / \mathrm{MAPBr}_{3}$ structure of cascade perovskite single crystal and heat it at $100^{\circ} \mathrm{C}$ for 6 hours. As shown in Figure S7c and Figure S7d, the chlorinebromide ions diffusion is obvious between $\mathrm{MAPbBr}_{2.5} \mathrm{Cl}_{0.5} / \mathrm{MAPCl}_{3}$. In contrast, the chlorinebromide is negligible between $\mathrm{MAPbBr}_{2.5} \mathrm{Cl}_{0.5} / \mathrm{MAPBr}_{3}$.

Based on these results, we found concentration gradient and lattice constant difference could both influence the interdiffusion speed in cascade OPSCs. For example, although the difference of halide ions concentration gradient is large between $\mathrm{MAPbBr}_{3} / \mathrm{MAPbI}_{3}$, the large difference between their lattice constant could limit the iodide-bromide ions interdiffusion; Although the lattice constant difference between $\mathrm{MAPbBr}_{2.5} \mathrm{Cl}_{0.5} / \mathrm{MAPBr}_{3}$ is small, the small concentration gradient could slow down the ions interdiffusion. The speed of chlorine-bromide ions interdiffusion is fast between the $\mathrm{MAPbBr}_{2.5} \mathrm{Cl}_{0.5}$ and $\mathrm{MAPCl}_{3}$ where the lattice constant is approaching (5.69 $\AA, 5.79 \AA$ ) and the concentration gradient difference is large.

As a result, to slow down the speed of chlorine-bromide interdiffusion, we use 6 layers to reduce the concentration gradient between adjacent layers. To slow down the speed of iodide-bromide interdiffusion, we could utilize the lattice constant difference between $\mathrm{MAPbI}_{3}$ and $\mathrm{MAPbBr}_{3}$. 


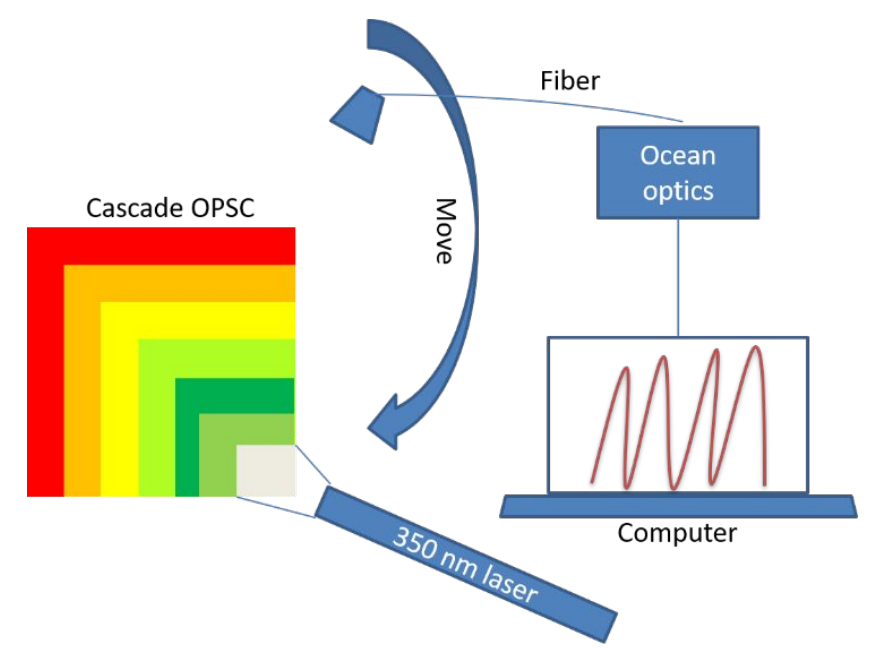

Figure S7. Assignment to measure the PL spectrum.

We use a $350 \mathrm{~nm}$ laser focus on the cross-section of the cascade OPSC. The photoluminescence emitted from cascade OPSC could be excited by two routes.

First route is the $350 \mathrm{~nm}$ laser directly excite the OPSCs with different halide compositions.

Second route is the photoluminescence of $\mathrm{MAPbCl}_{3}$ approaching $390 \mathrm{~nm}$ is first excited, then the $390 \mathrm{~nm}$ photons would then excite the photoluminescence of $\mathrm{MAPbBr}_{2} \mathrm{Cl}$ with $450 \mathrm{~nm}$ photons. Then the $450 \mathrm{~nm}$ photons would excite the photoluminescence of $\mathrm{MAPbBr}_{1.5} \mathrm{Cl}_{1.5}$ with $500 \mathrm{~nm}$. Then the $500 \mathrm{~nm}$ photons would excite the photoluminescence of $\mathrm{MAPbBr}_{2} \mathrm{Cl}$ with $550 \mathrm{~nm}$. Then the $550 \mathrm{~nm}$ photons would excite the photoluminescence of $\mathrm{MAPbBr}_{2.5} \mathrm{Cl}{ }_{0.5}$ with $570 \mathrm{~nm}$. Then the $570 \mathrm{~nm}$ photons would excite the photoluminescence of $\mathrm{MAPbBr}_{3}$ with $605 \mathrm{~nm}$. Then the 605 $\mathrm{nm}$ photons would excite the photoluminescence of $\mathrm{MAPbBr}_{2} \mathrm{I}$ with $720 \mathrm{~nm}$. As the bandgap becomes smaller with the halide composition variation, the photoluminescence starts from $390 \mathrm{~nm}$ $\left(\mathrm{MAPbCl}_{3}\right)$ and end with $720 \mathrm{~nm}\left(\mathrm{MAPbBr}_{2} \mathrm{I}\right)$. Finally, each layer of the cascade OPSC would be excited with photoluminescence.

Then, we use a fiber to collect the photoluminescence and the fiber is linked to Ocean optics (Maya 2000 Pro) for signal capture. Due to the light source consisted of 7 parts, we have to adjust the location of fiber until the spectrograph gets 7 peaks in one spectrum. 
As a result, the location of laser beam and fiber would both influence the intensity of photoluminescence with different wavelength. The data shown in Figure $2 \mathrm{c}$ is directly collected from the spectrograph. 


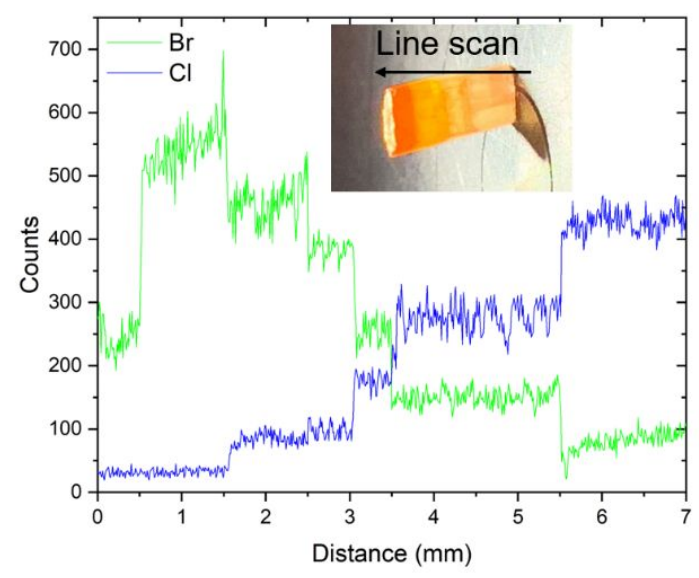

Figure S8. EDS line scan of the 7-layer cascade OPSC.

Due to the cascade OPSC used in Figure $2 \mathrm{~d}$ had been cut into the shape as shown in Figure $2 b$, the Figure S8 shows the line scan result of the 7-layer cascade OPSC. 

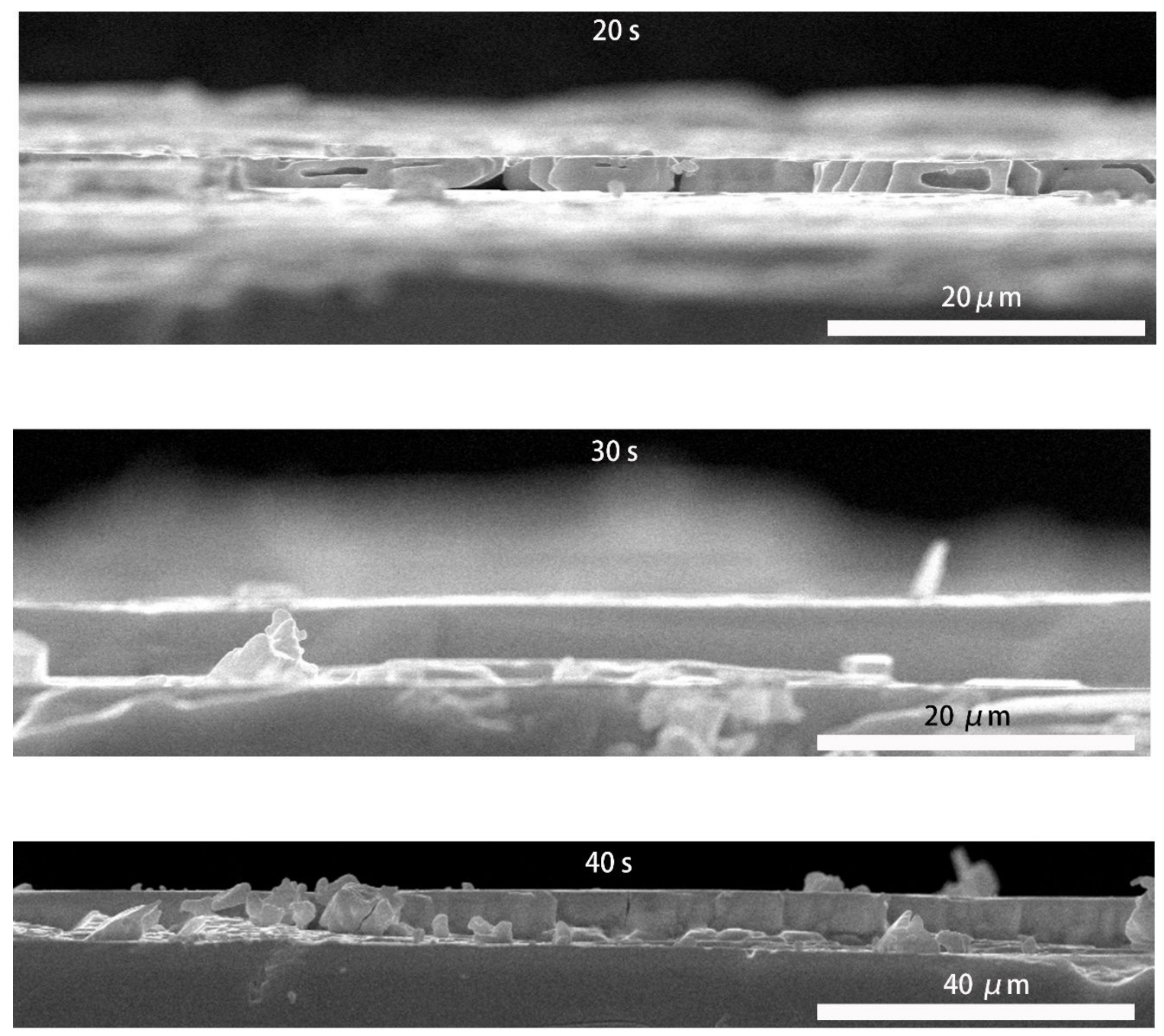

Figure S9. Thickness of SPE layer with different growth time. 

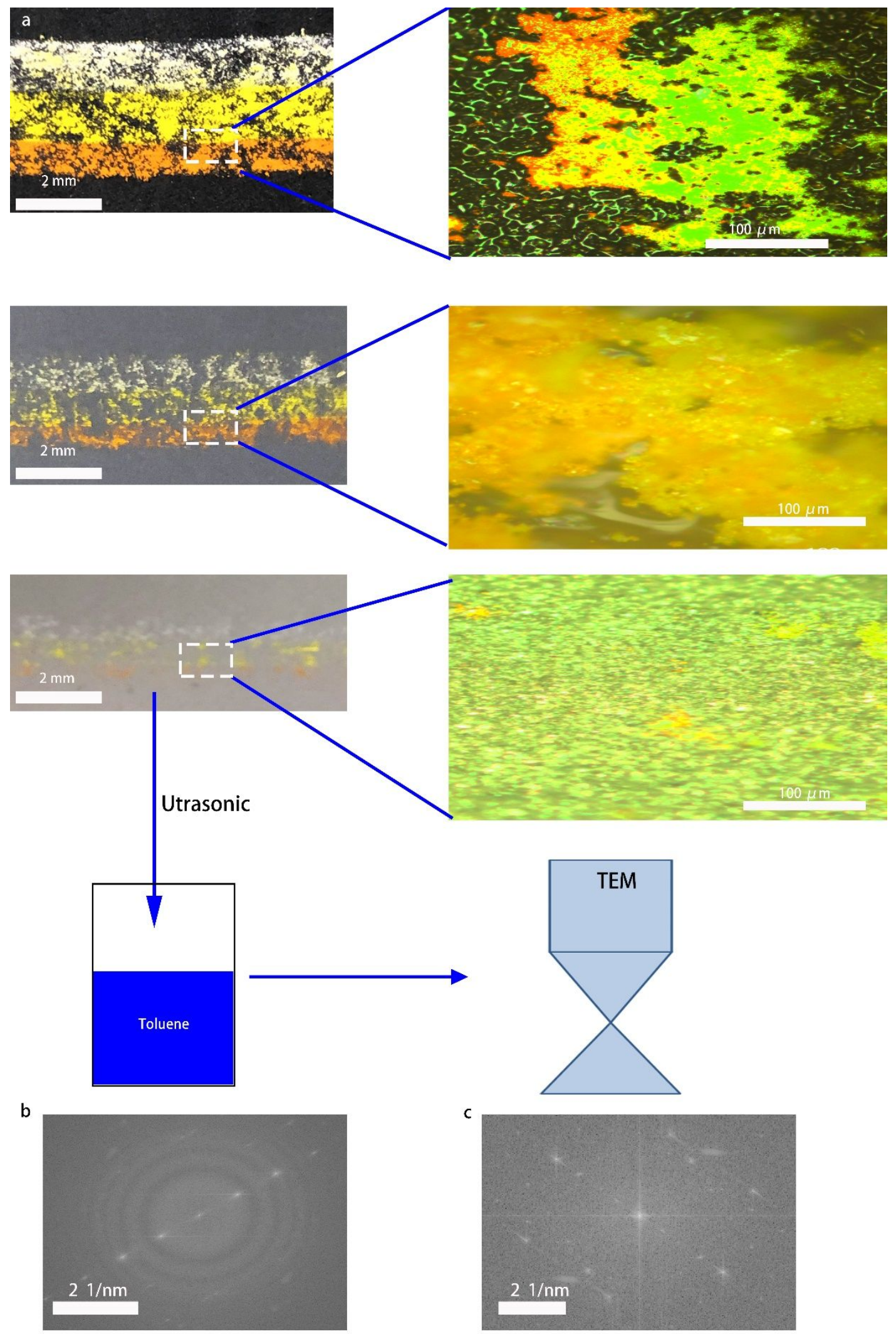

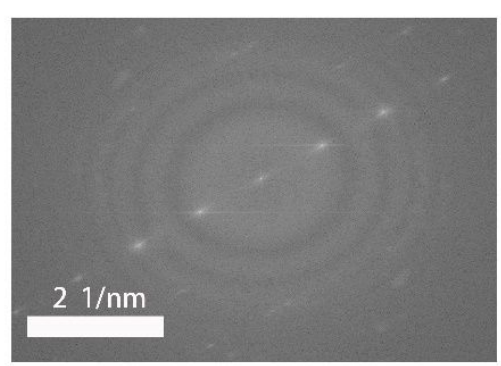

Figure S10. (a) Physical method to make tens of nanometer OPSC. (b)FFT transform of the HR-TEM image in Figure 3b. (c) FFT transform of the HR-TEM image in Figure 3c. 


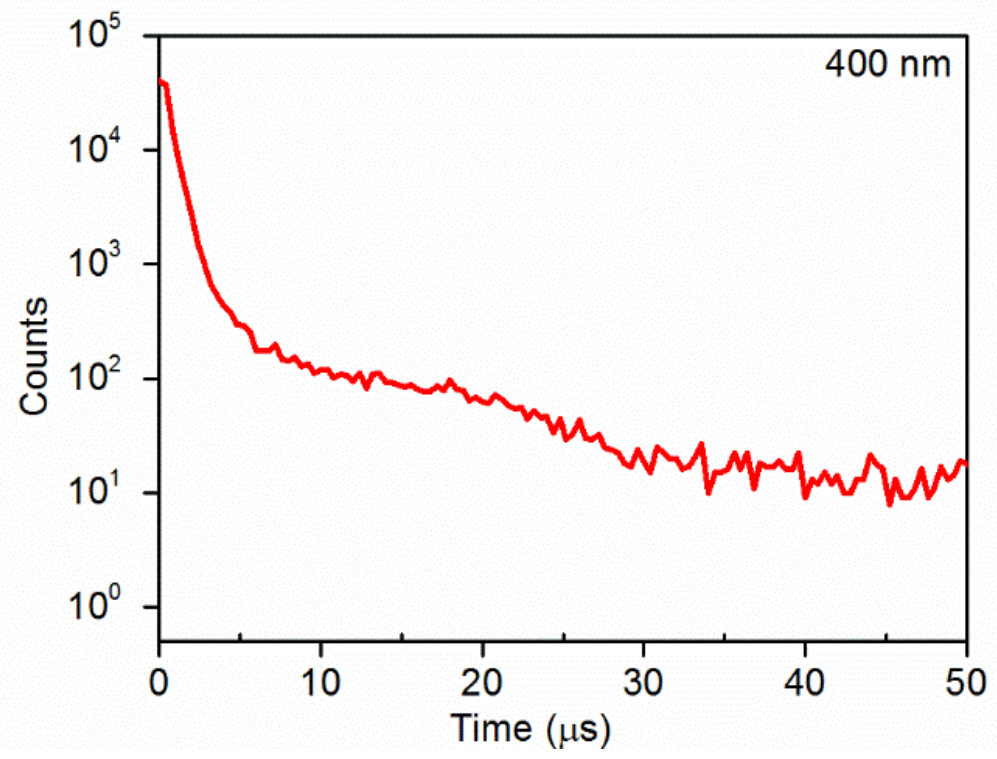

Figure S11. Time-resolved PL decay curve of the cascade OPSCs. Due to the laser is incident from the $\mathrm{MAPbCl}_{3}$ OPSC face, the PL peak located at $400 \mathrm{~nm}$. As a result, we chose $400 \mathrm{~nm}$ for the time-resolved PL decay experiment. 

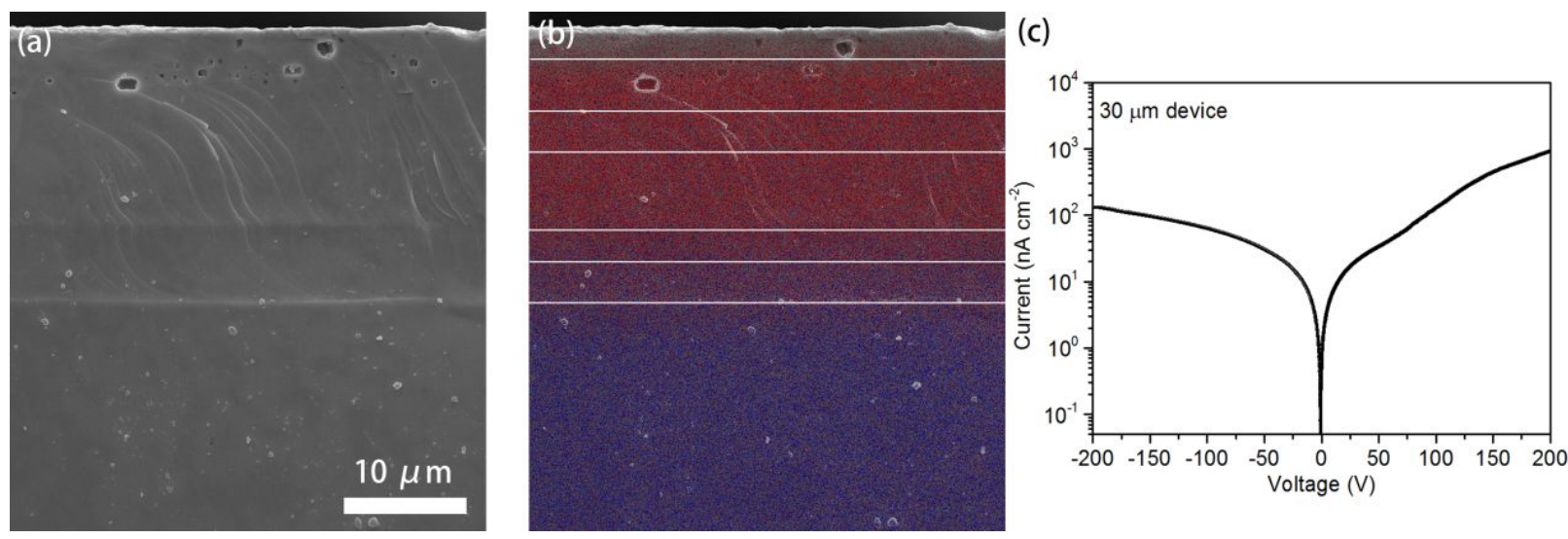

Figure S12. Cascade OPSCs that are $30 \mu \mathrm{m}$ thick. (a) Scanning electron microscopy image of a cross-section. (b) Elemental mapping results. Red is $\mathrm{Cl}$, and blue is $\mathrm{Br}$. (c) Currentvoltage curve of the diode constructed from the $30 \mu \mathrm{m}$-thick cascade OPSC. 

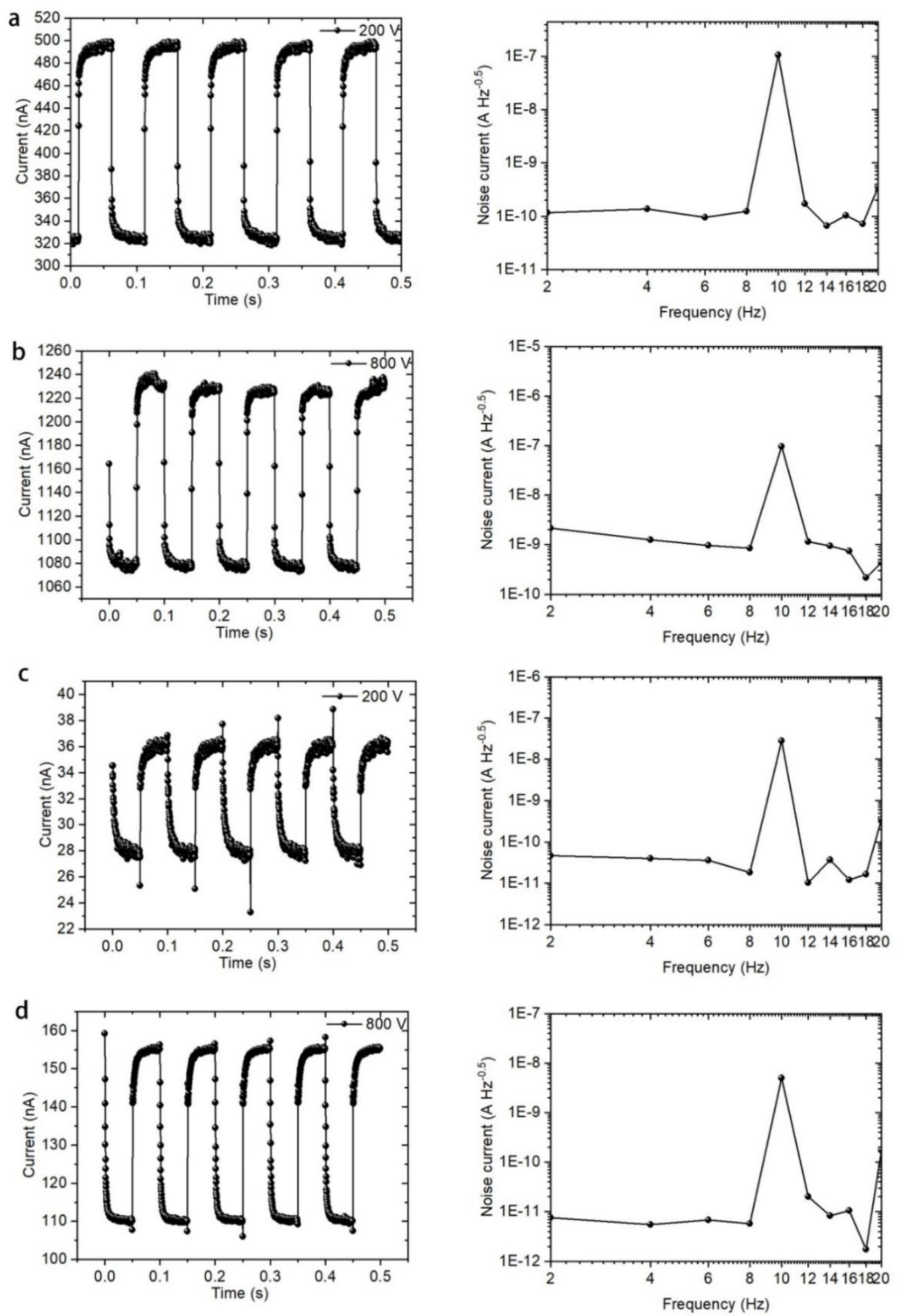

Figure S13. Noise current (Au/OPSC/Au vs Au/cascade OPSC/Au). (a) $\mathrm{Au} / \mathrm{MAPbBr}{ }_{2.5} \mathrm{Cl}_{0.5}$ OPSC/Au under $200 \mathrm{~V}$. (b) $\mathrm{Au} / \mathrm{MAPbBr}_{2.5} \mathrm{Cl}_{0.5} \mathrm{OPSC} / \mathrm{Au}$ under $800 \mathrm{~V}$. (c) $\mathrm{Au} /$ cascade OPSC/Au under 200V. (d) Au/cascade OPSC/Au under $800 \mathrm{~V}$.

Due to the direct X-ray detector require high electric filed to realize charges collection, we test the current noise under $200 \mathrm{~V}$ and $800 \mathrm{~V}$ respectively. Figure $\mathrm{S} 11 \mathrm{a}$ and Figure $\mathrm{S} 11 \mathrm{~b}$ is the noise current of $\mathrm{Au} / \mathrm{MAPbBr}_{2.5} \mathrm{Cl}_{0.5} \mathrm{OPSC} / \mathrm{Au}$ structure, Figure $\mathrm{S} 11 \mathrm{c}$ and Figure $\mathrm{S} 11 \mathrm{~d}$ is the noise current of $\mathrm{Au} /$ cascade $\mathrm{OPSC} / \mathrm{Au}$ structure. 

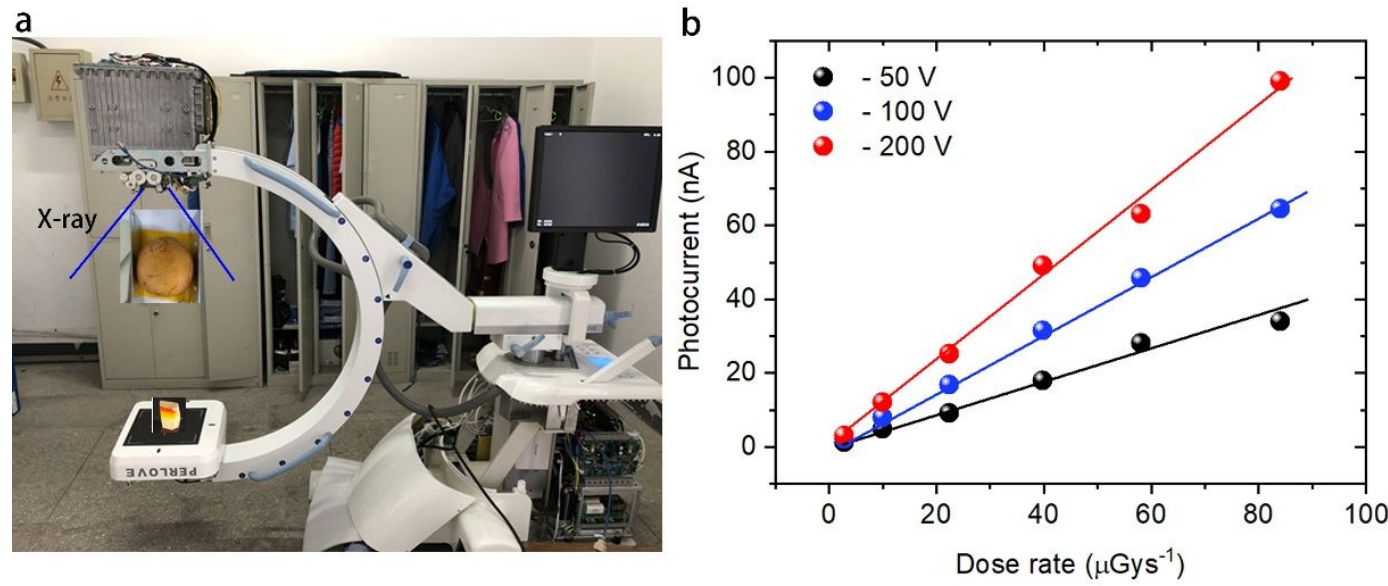

Figure S14. (a)Set-up of the X-ray detection experiment. (b)X-ray dose rate verse photocurrent under different voltage bias.

As shown in Figure $\mathrm{S} 11$, Areas of the contact is $2 \mathrm{~mm} * 4 \mathrm{~mm}$, the incident direction is shown in Figure S11. The dose rate is measured with commercial dosimeter (FJ-347A, China). The distance between X-ray tube and cascade OPSC is $1.2 \mathrm{~mm}$. The anode voltage and current are $140 \mathrm{kV}$ and $1 \mathrm{~mA}$ respectively. The temperature is $25^{\circ} \mathrm{C}$ when we did the experiments.

Following gives the dose rate with different anode current and anode voltage.

\begin{tabular}{|l|r|r|r|r|r|r|}
\hline $\begin{array}{c}\text { Anode } \\
\text { Current } \\
\text { Anode } \\
\text { Voltage } \\
(\mathrm{KV})\end{array}$ & 0.5 & 1 & 1.5 & 2 & & \\
\hline 80 & 1.82 & 7.64 & 14.66 & 22.45 & 32.13 & 45.43 \\
\hline 100 & 2.42 & 9.15 & 19.65 & 30.33 & 43.98 & 62.9 \\
\hline 140 & 2.84 & 10.02 & 22.02 & 37.96 & 55.33 & 79.63 \\
\hline 160 & 3.23 & 10.2 & 22.12 & 41.67 & 61.13 & 88.08 \\
\hline
\end{tabular}

Table S2. X-ray dose rate with different anode current and anode voltage 